\title{
Pharmacological Profile of JNJ-64179375: A Novel, Long-Acting Exosite-1 Thrombin Inhibitor
}

\author{
Zheng Huang Devine, Fuyong Du, Qiu Li, Matthew Bunce, Eilyn R. Lacy, \\ and Madhu Chintala \\ Cardiovascular \& Metabolism (Z.H.D., F.D., Q.L., M.B., M.C.) and Janssen Biotechnology (E.R.L.), Janssen Research \& \\ Development LLC, Spring House, Pennsylvania
}

Received June 25, 2019; accepted August 20, 2019

\begin{abstract}
JNJ-64179375 (JNJ-9375) is a recombinant human lgG4 monoclonal antibody engineered to mimic an $\lg A$ antibody that was identified in a patient who exhibited markedly prolonged clotting times but without spontaneous bleeding episodes over several years of follow-up. The crystal structure of the JNJ-9375 antigen-binding fragment/thrombin complex showed an almost identical binding mode to that of the patient $\lg A$. In the current study, we characterized the in vitro and in vivo properties of JNJ9375. Surface plasmon resonance studies demonstrated that JNJ-9375 binds to $\alpha$-thrombin with high affinity and specificity (K $K_{\mathrm{D}}: 0.8 \mathrm{nM}$ for human thrombin). JNJ-9375 produced concentration-dependent prolongation of in vitro clotting assays in human plasma, including thrombin time (TT), ecarin clotting time, prothrombin time, and activated partial thromboplastin time, with $\mathrm{EC}_{2 \times}$ values of $4.4,12.4,172.6$, and $202.7 \mu \mathrm{g} / \mathrm{ml}$, respectively. JNJ-9375 inhibited thrombin-induced platelet aggregation in human plasma with an $\mathrm{IC}_{50}$ value of $52.6 \mathrm{nM}(7.8 \mu \mathrm{g} / \mathrm{ml})$ and produced concentration-dependent prolongation of reaction time tested by thromboelastography. JNJ-9375 pretreatment resulted in dose-dependent reduction in thrombus
\end{abstract}

\section{Introduction}

Thrombin is a key serine protease involved in hemostasis and thrombosis (Furie and Furie, 2008). It is formed by proteolytic cleavage from prothrombin as the final step in the coagulation cascade. Thrombin plays a pivotal role in coagulation by mediating the conversion of soluble fibrinogen to insoluble fibrin that forms the meshwork of a clot. In addition, thrombin is also the most potent physiologic activator of platelets, contributing to the pathogenesis of arterial thrombosis (Sambrano et al., 2001). Thrombin has been an antithrombotic target since inhibition of its generation or function could help the management of cardiovascular thromboembolic disorders, which remain a major cause of morbidity and mortality.

The research was funded by Janssen Research \& Development LLC. https://doi.org/10.1124/jpet.119.261032. formation in the rat arteriovenous (AV) shunt model of thrombosis. Robust efficacy was observed at $0.3 \mathrm{mg} / \mathrm{kg}$ accompanied by $1.5 \times$ of $T$. Bleeding was increased at $3 \mathrm{mg} / \mathrm{kg}$ in a rat tail transection bleeding model demonstrating a therapeutic index of $10 \times$ compared with $1 \times$ for apixaban in the same models. Our data suggest that thrombin exosite I inhibition is efficacious against thrombosis in a pretreatment prevention animal model.

\section{SIGNIFICANCE STATEMENT}

JNJ-9375 is a novel, fully human monoclonal antibody that binds to the exosite I region of thrombin with high affinity and specificity. JNJ-9375 concentration dependently prolonged clotting times and inhibited thrombin-induced platelet aggregation in in vitro assays based on its mechanism of action. In an in vivo rat AV shunt model, JNJ-9375 prevented thrombus formation in a dose-dependent fashion while demonstrating reduced bleeding risk. The present study demonstrated the antithrombotic effects of inhibiting the exosite I region of thrombin when given in a prevention mode in preclinical animal models.

ABBREVIATIONS: aPTT, activated partial thromboplastin time; AV, arteriovenous; CAT, calibrated automated thrombogram; DOAC, direct-acting oral anticoagulant; ECT, ecarin clotting time; ETP, endogenous thrombin potential; JNJ-9375, JNJ-64179375; PAR1, proteinase-activated receptor1; PPP, platelet poor plasma; PRP, platelet rich plasma; PT, prothrombin time; TEG, thromboelastograph; TRAP-6, thrombin receptor agonist peptide-6; TT, thrombin time. 
of phase III trials of dabigatran, rivaroxaban, apixaban, and edoxoban in 71,683 patients with atrial fibrillation found a $19 \%$ decrease in stroke and systemic embolic events when using a DOAC compared with warfarin (Ruff, 2014)). A study of 17,701 patients undergoing major orthopedic surgery found that crude incidence of symptomatic thromboembolic events was $0.89 \%$ in the group receiving rivaroxaban for thromboprophylaxis, and $1.31 \%$ in the group treated with the standard of care. Treatment-emergent bleeding was similar between the two groups $(0.44 \%$ and $0.33 \%$, respectively) (Turpie, 2014). DOACs are also more convenient to administer and do not require routine coagulation monitoring. However, they are still associated with some bleeding risk at their effective therapeutic doses, especially with direct thrombin inhibitors (Weitz and Jaffer, 2016; Weitz and Harenberg, 2017). As a result, there are still a substantial number of patients at risk of bleeding that are being underdosed or not treated (Barra et al., 2016; Xian et al., 2017).

JNJ-64179375 (JNJ-9375) is a fully humanized recombinant IgG4 that reversibly binds to the exosite I region of thrombin with high affinity and specificity. JNJ-9375 was engineered to mimic an IgA antibody that was identified in a patient with markedly prolonged clotting times [thrombin time (TT) unclottable with human thrombin, prothrombin time (PT) prolongation of more than three times, and activated partial thromboplastin time (aPTT) prolongation of more than seven times] but without spontaneous bleeding over a prolonged follow-up period (Baglin et al., 2016). the IgA bind the exosite I domain and inhibit the binding of fibrinogen to thrombin Fig 1, which consequently prevents the conversion of soluble fibrinogen to insoluble fibrin in forming the framework of a clot (Huntington, 2013). In addition, JNJ-9375 prevents the binding of other substrates to exosite I on thrombin, including proteinase-activated receptor-1 (PAR1) on platelets and the anticoagulant thrombomodulin. This mechanism is distinct from direct thrombin inhibitors on the market, such as dabigatran and bivalirudin, since it spares the function of the active catalytic site and exosite II (Eisert et al., 2010). Therefore, JNJ-9375 has the potential to have a different phenotypic profile due to differential interaction with its various substrates, and thereby may achieve a distinct anticoagulation profile with reduced bleeding risk compared with available drugs.

In the current study, we characterized the in vitro pharmacological profile of JNJ-9375, including its interaction with thrombin and effect on clotting times, platelet aggregation, and thrombin generation. In addition, we tested the antithrombotic efficacy of JNJ-9375 in vivo in a rat arteriovenous (AV) shunt model of thrombosis. Apixaban, a marketed factor $\mathrm{Xa}$ inhibitor, was also evaluated as a comparator.

\section{Materials and Methods}

Drugs and Reagents. JNJ-9375, control human IgG4 (CNTO 3161), and buffer were obtained internally (Janssen Biotherapeutics, Malvern, PA). Apixaban was obtained from Selleckchem (Houston, TX). Human $\alpha$-thrombin and prothrombin were obtained from either Haematologic Technologies (Essex Junction, VT) or Enzyme Research Laboratories (South Bend, IN). Mouse and rat thrombin were obtained from Enzyme Research Laboratories. Cynomolgus thrombin, meizothrombin variant $\mathrm{R} 155 \mathrm{Q} / \mathrm{R} 271 \mathrm{Q} / \mathrm{R} 284 \mathrm{Q}$ (referred to as meizothrombin QQQ), and meizothrombin variant R155Q/R271Q/R284Q/S195A (referred to as meizothrombin QQQA) were recombinantly expressed and obtained from Cambridge Protein Works (Cambridge, UK). D-Phenylalanyl-prolyl-arginyl chloromethyl ketone-inactivated human $\alpha$-thrombin was prepared internally. Coagulation reagents were from Diagnostica Stago (Parsippany, NJ): Thrombin 10 for TT, ecarin for ecarin clotting time (ECT), Neoplastine Cl Plus 5 for PT, and C.K. Prest 5 and $\mathrm{CaCl}_{2}$ for aPTT. For the platelet aggregation analysis, $\mathrm{ADP}$, collagen, and arachidonic acid were obtained from Chronolog (Havertown, PA); thrombin receptor agonist peptide-6 (TRAP-6) and H-Gly-Pro-Arg-Pro-OH-AcOH were obtained from Bachem (King of Prussia, PA).

Biacore Experiment for Substrate Affinity Determination. Surface plasmon resonance analysis was performed using a BiacoreT200 instrument [GE healthcare, Uppsala, Sweden (previously Biacore $\mathrm{AB}$ )]. The experiments were performed in PBS buffer ( $\mathrm{pH} 7.4$, with $3 \mathrm{mM}$ EDTA, $0.005 \%$ Tween 20 , and $400 \mu \mathrm{g} / \mathrm{ml}$ bovine serum albumin). Goat anti-human Fc $\gamma$ antibody (Jackson ImmunoResearch, West Grove, PA) was covalently immobilized onto a CM5 sensor chip per the manufacturer's instructions. Approximately 13,000 response units of anti-human Fc $\gamma$ antibody were covalently immobilized in a reaction and reference flow cell. The remaining reactive groups on the surface were deactivated by injection of ethanolamine-HCl. JNJ9375 or CNTO 3161 were captured onto the anti-Fc $\gamma$-modified sensor chip (120-140 response units). The antibody capture was followed by injection of serially diluted possible binding substrates (human, cynomolgus monkey, mouse, rat thrombin, prothrombin, or meizothrombin) at $40 \mu \mathrm{l} / \mathrm{min}$. To perform the kinetic experiments, the antigens were prepared in the PBS-based buffer described previously, following a 2-fold serial dilution to obtain a concentration range of 0.039-20 nM, and the interactions were monitored at $25^{\circ} \mathrm{C}$. Regeneration of the anti-Fc surface after each cycle was achieved using $10 \mathrm{mM}$ glycine ( $\mathrm{pH} 2.0$ ). The data collected were processed using the T200 evaluation software (version 2) and fitted to a Langmuir 1:1 binding model, with the results reported in the format of $k_{\text {on }}$ (on rate), $k_{\text {off }}$ (off rate), and $K_{\mathrm{D}}$ (equilibrium dissociation constant).

Coagulation Assays. Assays were run on citrated plasma from the blood of healthy volunteers obtained via antecubital vein. All of the blood donors were required to be at least 2 weeks free of any medications having anticoagulant or antiplatelet effects, which included nonsteroidal anti-inflammatory drugs, warfarin, dabigatran, apixaban, and rivaroxaban. Rat blood was collected from the abdominal aorta and mouse blood was collected from the posterior vena cava. Male Sprague-Dawley rats (350-450 g) were purchased from Charles River Laboratories (Frederick, MD), and male C57 black mice (20-30 g) were purchased from Taconic Biosciences (Hudson, NY). Blood was mixed with $3.2 \%$ sodium to make a $1: 10$ dilution and centrifuged at $2500 \mathrm{~g}$ for 15 minutes at $4^{\circ} \mathrm{C}$. The resulting platelet poor plasma (PPP) was immediately stored at $-80^{\circ} \mathrm{C}$ until use. Cynomolgus monkey and rabbit PPP were purchased from BioreclamationIVT (Westbury, NY).

Standard clotting assays including TT, ECT, PT, and aPTT were performed in plasma samples spiked with JNJ-9735 as described previously (Douxfils et al., 2012), or from ex vivo plasma samples obtained from rats that were subjected to the AV shunt model of thrombosis. Coagulation assays were performed on a four-channel coagulometer STA rt4 (Diagnotica Stago). Briefly, for the TT assay, $100 \mu \mathrm{l}$ PPP containing different concentrations of JNJ-9375 was incubated for 1 minute at $37^{\circ} \mathrm{C}$ in a cuvette and coagulation was initiated by adding $100 \mu \mathrm{l}$ thrombin [1.5 U/ml (National Institutes of Health units)]. The onset of clotting was recorded as the coagulation time. For the ECT assay, $100 \mu \mathrm{l}$ PPP containing different concentrations of JNJ-9375 was incubated for 4 minutes at $37^{\circ} \mathrm{C}$ in a cuvette and coagulation was initiated by adding $50 \mu \mathrm{l}$ ecarin $(5 \mathrm{U} / \mathrm{ml}$, reconstituted in $\mathrm{H}_{2} \mathrm{O}$ ). For the PT assay, $50 \mu$ l PPP containing different concentrations of JNJ-9375 was incubated for 1 minute at $37^{\circ} \mathrm{C}$ in a cuvette and coagulation was initiated by adding $100 \mu \mathrm{l}$ Neoplastine $\mathrm{Cl}$ reagent. For the aPTT assay, $50 \mu \mathrm{l} \mathrm{C.K.} \mathrm{Prest} \mathrm{reagent}$ was incubated with $50 \mu \mathrm{l}$ PPP containing different concentrations of $\mathrm{JNJ}-9375$ for 3 minutes at $37^{\circ} \mathrm{C}$ in a cuvette and coagulation was 
A

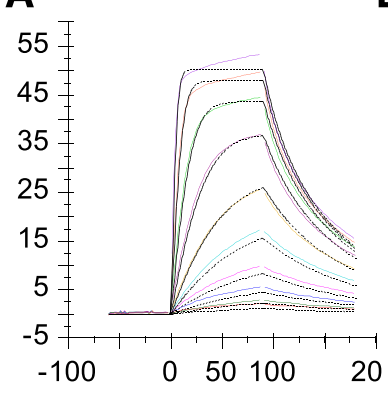

B

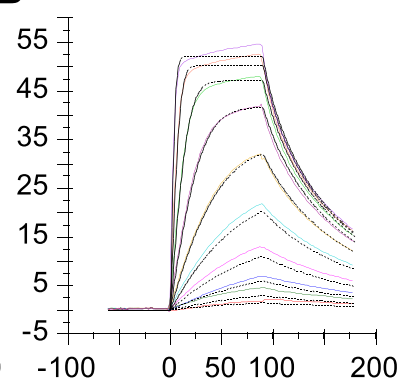

C

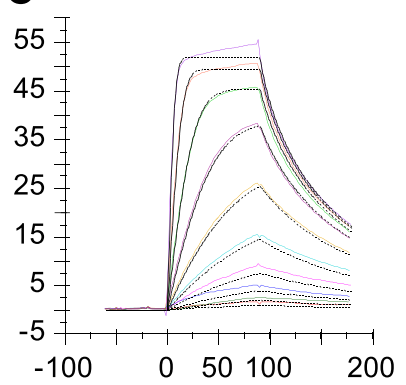

E
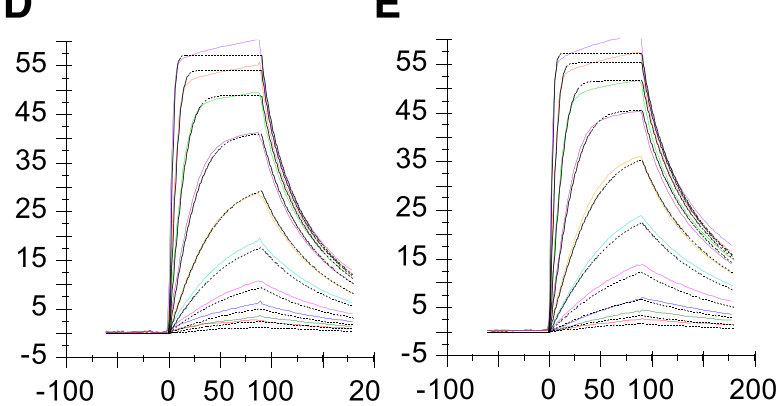

$\mathbf{F}$

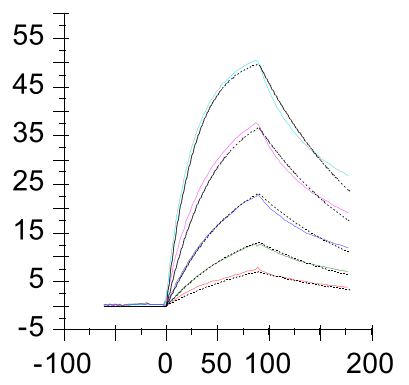

G

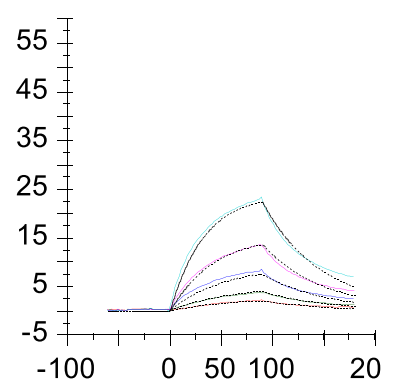

H

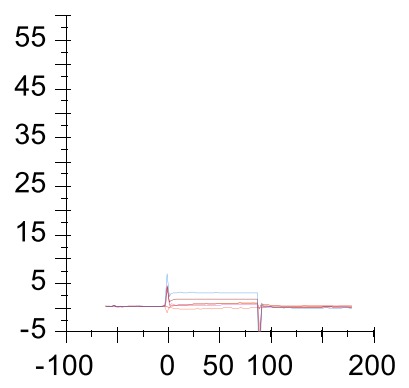

I

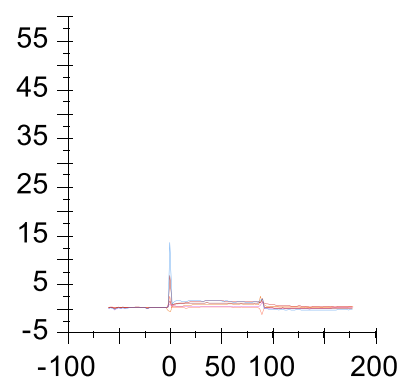

Fig. 1. Representative Biacore sensorgrams for the interaction of JNJ-9375 with $\alpha$-thrombin/D-phenylalanyl-prolyl-arginyl chloromethyl ketone (A), human $\alpha$-thrombin (B), cynomolgus monkey thrombin (C), rat thrombin (D), mouse thrombin (E), meizothrombin variant $\mathrm{R} 155 \mathrm{Q} / \mathrm{R} 271 \mathrm{Q} / \mathrm{R} 284 \mathrm{Q} /$ $\mathrm{S} 195 \mathrm{~A}$ (F) meizothrombin variant $\mathrm{R} 155 \mathrm{Q} /$ $\mathrm{R} 271 \mathrm{Q} / \mathrm{R} 284 \mathrm{Q}(\mathrm{G})$, and prothrombin $(\mathrm{H})$. Also shown is CNTO 3161 with prothrombin (I). All other data for the negative control antibody looked similar to (I). These data were obtained in PBS-based buffer using Biacore T200.

\section{Time (s)}

initiated by adding $50 \mu \mathrm{l} 25 \mathrm{mM} \mathrm{CaCl}_{2}$. The ratio change in clotting time induced by JNJ-9375 was calculated as the fold increase over the baseline value from the vehicle control group.

Platelet Aggregation. Citrated blood, as in the clotting test, was processed to yield platelet rich plasma (PRP) by centrifuging the blood samples at $160 \mathrm{~g}$ for $10-12$ minutes at room temperature. The resultant $\mathrm{PRP}$ was transferred to $15 \mathrm{ml}$ falcon tubes. The remaining samples were then further centrifuged at $2500 \mathrm{~g}$ for 15 minutes at room temperature to collect PPP. The PRP was adjusted to a platelet count of $300 \times 10^{3} / \mu \mathrm{l}$ with addition of the PPP from the donor. The PRP was kept at room temperature until use and used within 3 hours of preparation. Rat PRP and PPP were prepared using the same protocol except that the blood was drawn from the abdominal aorta and the final PRP platelet count was adjusted to $600 \times 10^{3} / \mu$ l (Derian et al., 1995).

Platelet aggregation was measured using the 700 Whole Blood/ Optical Lumi-Aggregometer from the Chrono-log Corporation. Platelet aggregation was determined by measuring the optical density change of stirred PRP after adding the aggregating agonist (Zhou and Schmaier, 2005; Hawes et al., 2015). Briefly, $250 \mu$ l of PRP was added to a cuvette with a stir bar and prewarmed at $37^{\circ} \mathrm{C}$ on the aggregometer sample storage area for at least 5 minutes. JNJ-9375 was added to the cuvette for pretreatment and incubated for 5 minutes before adding agonist. When thrombin was used as the agonist, PRP was pretreated with H-Gly-Pro-Arg-Pro-OH-AcOH (3 mM final concentration) for 2-4 minutes to prevent fibrin polymerization. The cuvette with PPP was then placed in the background chamber to represent $100 \%$ light transmittance. The PRP test cuvettes were transferred to the test chamber and the test was started by adding the corresponding agonists. The reaction was allowed to run for 5 minutes. Platelet aggregation led to an increase in the light transmittance, which was recorded and the percentage of aggregation was determined as the highest point of deflection from the baseline. The inhibition of platelet aggregation was determined by comparing the aggregation responses in the control, vehicle-treated, and antagonisttreated samples.

Thrombin Generation Assay. Thrombin generation was measured in PPP according to the Thrombinoscope guidelines using a calibrated automated thrombogram (CAT) system (Diagnostica Stago). Briefly, $80 \mu \mathrm{l}$ of citrated PPP was mixed with $20 \mu \mathrm{l}$ PPP reagent in round-bottom 96 -well plates and thrombin generation was initiated after 10 -minute preincubation at $37^{\circ} \mathrm{C}$ by adding $20 \mu \mathrm{l}$ of FluCa reagent (Diagnostica Stago). Fluorescence (excitation: $360 \mathrm{~nm}$; emission: $460 \mathrm{~nm}$ ) was measured every 20 seconds for 60 minutes on a Fluoroskan Ascent plate reader (Thermo) and thrombin concentration was determined by the software using a thrombin calibrator reagent. Parameters including lag time, peak thrombin, and endogenous thrombin potential (ETP) 
were derived and plotted as a function of thrombin inhibitor concentration.

Thromboelastograph. Thromboelastograph (TEG) analysis was performed with TEG-5000 analyzers (Haemonetics, Braintree, MA) following modification of the citrated RapidTEG protocol. Briefly, freshly drawn citrated blood from healthy volunteers was mixed with test compound or buffer control and incubated at $25^{\circ} \mathrm{C}$ for 5 minutes. The blood $(340 \mu \mathrm{l})$ was transferred into a TEG sample cup preloaded with $20 \mu \mathrm{l}$ of $0.2 \mathrm{M}$ calcium chloride and $10 \mu \mathrm{l}$ of RapidTEG reagent diluted 1:10 in HEPES-buffered saline [20 mM HEPES and $150 \mathrm{mM}$ $\mathrm{NaCl}$ ( $\mathrm{pH}$ 7.4)], and the TEG recording was initiated immediately. The 1:10 dilution of RapidTEG used was determined for optimal reproducibility of TEG parameters between multiple rounds of analysis. Two TEG-5000 analyzers (four channels) were in operation simultaneously for each titration series, with one channel used as a buffer control during each measurement. The interday and interchannel variability was minimal and met the standard set by the manufacturer (data not shown). From the TEG output, three key parameters, the reaction time, maximal amplitude, and percentage of lysis at 30 minutes after maximal amplitude, were extracted to assess clotting time, clot strength, and fibrinolytic activity, respectively.

Animals. Male Sprague-Dawley rats (350-450 g) were used for the in vivo studies. All animal care and experimental procedures were approved by the Institutional Animal Care and Use Committee at Janssen Research \& Development, LLC, and all animal studies were conducted in accordance with the Guide for the Care and Use of Laboratory Animals.

Rat AV Shunt Model of Thrombosis. JNJ-9375 and apixaban were evaluated on the antithrombotic efficacy in the rat AV shunt model. Rats were anesthetized by intramuscular administration of $100 \mathrm{mg} / \mathrm{kg}$ of ketamine plus $10 \mathrm{mg} / \mathrm{kg}$ of xylazine (Hanna's Pharmaceutical Supply CO., Wilmington, DE). Rats were placed on a heating pad to maintain body temperature and allowed to breathe normally on their own. Surgery was performed to isolate and catheterize the right carotid artery and both jugular veins to allow for administration of drugs, blood collection, and induction of thrombosis as previously described (Shang et al., 2014). After collecting a baseline blood sample
( $1 \mathrm{ml}$ ), drugs or vehicle were administered to the animals as an intravenous bolus 15 minutes before induction of thrombosis. An external tube was connected from the right carotid artery to the left jugular vein to initiate thrombi formation. Briefly, the shunt was prepared from a $6 \mathrm{~cm}$ section of Tygon tubing (Cole Parmer, Vernon Hills, IL) that connects on one end to the right carotid artery cannula and on the other end to the left vein cannula with $8 \mathrm{~mm}$ of special Masterflex Tygon tubing. A $5 \mathrm{~cm}$ length of surgical silk (size 2-0) (Ethicon, Bridgewater, NJ) was secured inside the shunt. All of the pieces of the shunt (except the 2-0 silk tread) were siliconized at least 24 hours prior to the assembly. Blood was allowed to flow through the tubing for 15 minutes and a blood clot was allowed to form on the thread. At the end of 15 minutes, the external tubing with the thread and blood clot was removed and the blood clot was weighed. A second blood sample $(5 \mathrm{ml})$ was collected from the abdominal aorta and the animal was euthanized while still under anesthesia. Blood samples were used for drug level measurement and clotting assays.

Rat Tail Transection Bleeding Model. JNJ-9375 (0.3, 1, 3, and $10 \mathrm{mg} / \mathrm{kg})$ and apixaban $(0.3,1$, and $3 \mathrm{mg} / \mathrm{kg})$ were tested in a rat tail transection model to evaluate bleeding risks. Five minutes after receiving the intravenous injection of compounds, the rat tails were transected at a diameter of $3 \mathrm{~mm}$ and the cut ends of the tails were immediately immersed into $50-\mathrm{ml}$ tubes filled with $40 \mathrm{ml}$ of prewarmed saline. Bleeding was continuously monitored, and the tube was changed with prewarmed saline every 5 minutes for a total of 30 minutes. The readouts included the time to stop bleeding, total bleeding time, and total blood loss determined by measuring the hemoglobin mass in the saline using the QuantiChrom hemoglobin assay kit in which hemoglobin is converted into a colored end product and the intensity of the color is proportional to the hemoglobin concentration.

JNJ-9375 Concentration Measurement. Measurement of the JNJ-9375 pharmacokinetic concentration in rat plasma was determined using an immunoassay method employing a homogenous sandwich assay format with electrochemiluminescent detection. The capture and detection reagents in the assay were both anti-human Fc monoclonal antibodies. Rat plasma samples were analyzed in the
A

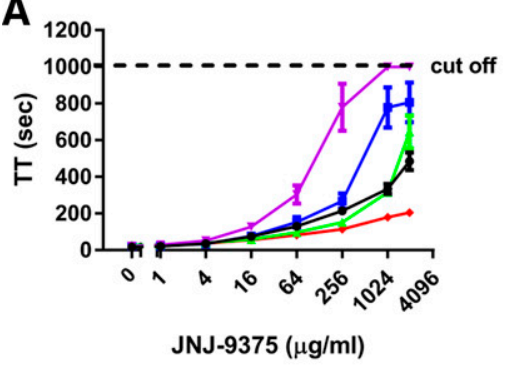

D

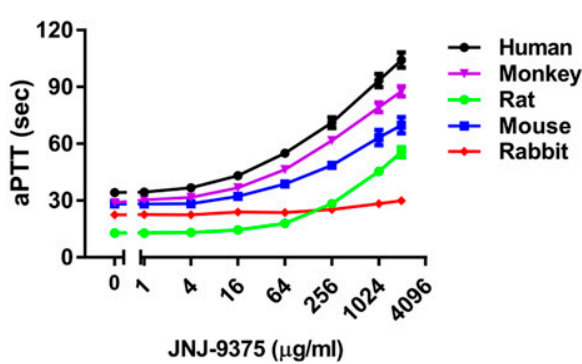

B

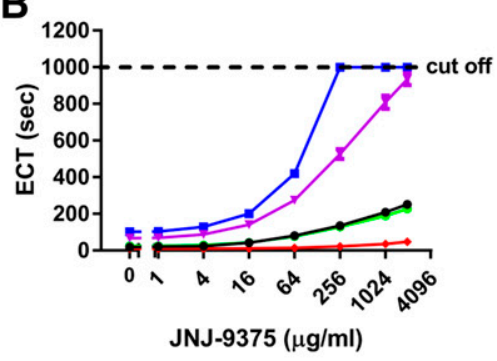

E

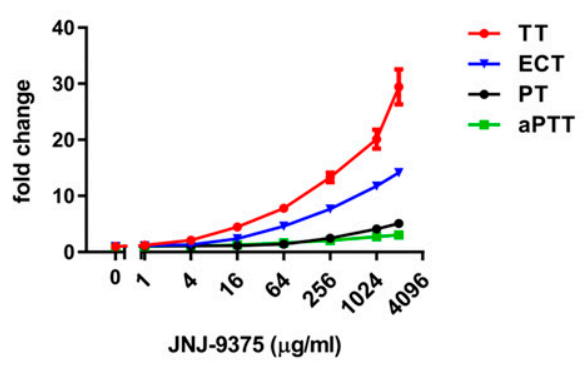

C

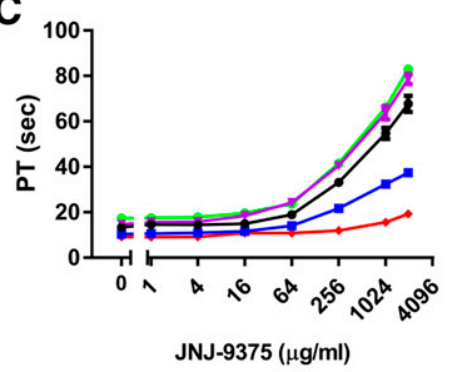

$\mathbf{F}$

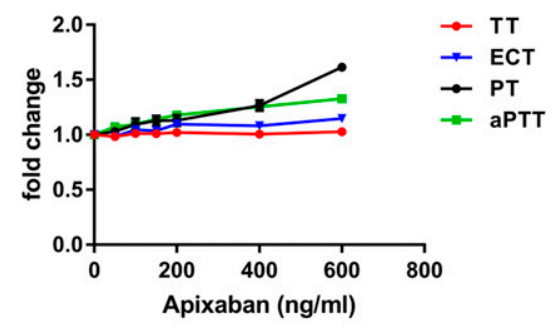

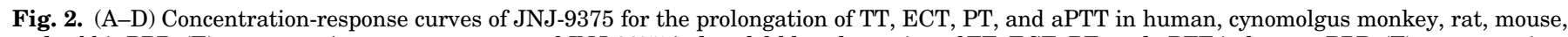

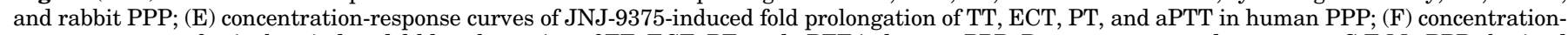

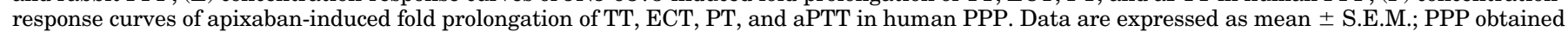
from four to six donors in each species. 
assay at 1/500 dilution. Briefly, diluted standards, quality controls, and samples containing the analyte were combined with a master mix containing the final working concentrations of the capture and detection reagent and incubated for 45 minutes at room temperature. The Meso Scale Discovery plate was washed and read on the Meso Scale Discovery sector imager (Meso Scale Diagnostics, Rockville, MD). The Meso Scale Discovery output files with the raw electrochemiluminescent counts were swept into Cyberlab and imported into Watson LIMS regression software for analysis. The Watson study regression used a five-parameter logistic (autoestimate) fit with a $1 / Y^{2}$ weighting factor.

Data Analysis and Statistics. All of the data are expressed as mean \pm S.E.M. Statistical analyses were performed using GraphPad Prism software (version 6.0; La Jolla, CA). Significance was defined as $P<0.05$ as determined by one-way ANOVA followed by Tukey's or Dunnett's post hoc test for multiple comparisons. R package nlme was used to calculate the $\mathrm{EC}_{2 \mathrm{x}}$ values for in vitro clotting assays using the polynomial mixed-effects model.

\section{Results}

In Vitro Binding Assays. Binding experiments were performed on a Biacore to determine the affinity of JNJ9375 to human, cynomolgus monkey, mouse, and rat thrombin as well as to investigate the crossreactivity of JNJ-9375 with prothrombin and meizothrombin. Figure 1 shows an example of sensorgrams. The data were fitted to a 1:1 binding model and the results are summarized in Table 1. Our data showed that JNJ-9375 bound to human, cynomolgus monkey, rat, and mouse thrombin with $K_{\mathrm{D}}$ values of $0.8 \pm 0.1,1.0 \pm 0.06,1.2 \pm$ 0.06 , and $0.8 \pm 0.04$, respectively. JNJ-9375 showed little to no binding to prothrombin (Table 1). However, JNJ-9375 bound to the protease active mutant $\mathrm{QQQ}$ (resistant to autolysis and remained in the active state) and the meizothrombin protease dead mutant QQQA (Ala replacing Ser in the catalytic domain; catalytically inactive but stable) (Bradford and Krishnaswamy, 2012) with moderate but comparable affinity $\left(K_{\mathrm{D}}\right.$ values of 80 and $11 \mathrm{nM}$, respectively), suggesting the protease activity is not required for JNJ-9375 binding. The negative control monoclonal antibody CNTO 3161 did not bind to thrombin (Fig. 2I). The data also showed that JNJ-9375 binds to the human $\alpha$-thrombin/inhibitor complex (human $\alpha$-thrombin/D-phenylalanyl-prolyl-arginyl chloromethyl ketone) with similar affinity than the inhibitor-free human

TABLE 1

Biacore affinity data for JNJ-9375 obtained by kinetic analysis in PBS buffer

\begin{tabular}{lccc}
\hline Analyte & $k_{\text {on }} \times 10^{7}$ & $k_{\text {off }} \times 10^{-2}$ & $K_{\mathrm{D}}{ }^{a}$ \\
\hline & $1 / M s$ & $1 / s$ & $n M$ \\
Human $\alpha$-thrombin/PPACK & $1.98 \pm 0.65$ & $3.02 \pm 0.82$ & $1.7 \pm 0.69$ \\
Human $\alpha$-thrombin & $3.28 \pm 0.16$ & $2.65 \pm 0.20$ & $0.8 \pm 0.10$ \\
Cynomolgus monkey & $3.06 \pm 0.22$ & $3.11 \pm 0.24$ & $1.0 \pm 0.06$ \\
thrombin & & & \\
Meizothrombin QQQ & & & \\
Meizothrombin QQQA & & & \\
Mouse thrombin & $0.05 \pm 0.04$ & $1.92 \pm 0.13$ & $79.9 \pm 67.1$ \\
Rat thrombin & $4.27 \pm 0.09$ & $1.17 \pm 0.37$ & $11.2 \pm 6.37$ \\
Prothrombin & $3.68 \pm 0.11$ & $4.54 \pm 0.20$ & $0.8 \pm 0.04$ \\
& & & $1.2 \pm 0.06$ \\
\hline
\end{tabular}

PPACK, D-phenylalanyl-prolyl-arginyl chloromethyl ketone.

${ }^{a}$ The average $K_{\mathrm{D}}$ value was calculated as an average of the individual $K_{\mathrm{D}}$ values. The averages were derived from four to 12 replicates.

${ }^{b}$ This is meizothrombin variant R155Q/R271Q/R284Q.

${ }^{c}$ This is meizothrombin variant R155Q/R271Q/R284Q/S195A.

${ }^{d}$ Little or no binding was observed and there was not enough kinetic information for affinity determination. The $K_{\mathrm{D}}$ value is expressed as more than the highest concentration of prothrombin injected. $\alpha$-thrombin (Table 1), confirming that protease activity is not required for JNJ-9375 binding.

Effect of JNJ-9375 on Clotting Times. JNJ-9375 produced concentration-dependent prolongation of TT, ECT, aPTT, and PT clotting times in plasma from all species studied (Fig. 3, A-D; Table 2). The JNJ-9375 concentrations required for doubling of the in vitro coagulation times in different species are shown in Table 2. Of the four coagulation assays tested, TT was the most sensitive assay to show the pharmacodynamic activity of JNJ-9375 (Fig. 3E), since the $\mathrm{EC}_{2 \mathrm{X}}$ values for ECT, PT, and aPTT were 2.8, 39.2, and 46.1 times higher than that of TT, respectively. ECT was also markedly prolonged by JNJ-9375, which demonstrates that JNJ-9375 inhibits the activity of endogenous meizothrombin and thrombin in the plasma. Modest changes induced by JNJ-9375 were also observed in the PT and aPTT assays. In contrast, apixaban, a marketed factor Xa inhibitor, exhibited minor concentration-dependent prolongation on PT and aPTT and no effects on TT and ECT when tested at concentrations covering that of clinical range (19-162 ng/ml) (Fig. 3F), which is consistent with its mechanism of action and clinical observations.

Effect of JNJ-9375 on Platelet Aggregation. The effect of JNJ-9375 on platelet aggregation was tested in PRP since the PAR1 on platelet surface is another important binding site of exosite I. JNJ-9375 produced potent, concentrationdependent inhibition of platelet aggregation in human PRP induced by $30 \mathrm{nM} \alpha$-thrombin. The aggregation (Fig. 4A, illustrated as deflection from the baseline) was decreased from $75.8 \% \pm 1.1 \%$ in the vehicle group to $0.5 \% \pm 0.3 \%$ in the 1000 nM JNJ-9375 group, with an IC $_{50}$ value of $52.6 \mathrm{nM}(7.8 \mu \mathrm{g} / \mathrm{ml})$ (Fig. 4B). Control human IgG4 at $1000 \mathrm{nM}$ did not inhibit thrombin-induced aggregation. JNJ-9375 at $100 \mathrm{nM}$ had no inhibitory effect on platelet aggregation induced by $\mathrm{ADP}$ (20 $\mu \mathrm{M})$, TRAP-6 (15 $\mu \mathrm{M})$, collagen $(3 \mu \mathrm{g} / \mathrm{ml})$, and arachidonic acid (1 mM) (Fig. 4C). However, aggregation induced by ADP, TRAP-6, and collagen were blocked by cangrelor (ADP antagonist), vorapaxar (PAR1 antagonist), and aspirin (cyclooxygenase inhibitor), respectively (data not shown).

The effect of JNJ-9375 on platelet aggregation was also tested in rat PRP using human $(80 \mathrm{nM})$ and rat $\alpha$-thrombin $(60 \mathrm{nM})$ as agonists. The concentrations of thrombin were the minimum dose to elicit aggregation based on our preliminary data. As shown in Fig. 4, D and E, both rat and human $\alpha$-thrombin led to robust aggregation $(73 \%-74 \%)$ in rat PRP. Pretreatment with JNJ-9375 significantly decreased thrombin-induced platelet aggregation at $100 \mathrm{nM}$ and completely inhibited platelet aggregation at 300 and $1000 \mathrm{nM}\left[\mathrm{IC}_{50}\right.$ values of $113 \mathrm{nM}(16.9 \mu \mathrm{g} / \mathrm{ml})$ and $86.9 \mathrm{nM}$ (13 $\mu \mathrm{g} / \mathrm{ml})$ ] when rat and human thrombin were used, respectively). The control IgG4 (1000 $\mathrm{nM})$ did not have any effect on thrombin-induced platelet aggregation.

Effect of JNJ-9375 on Thrombin Generation in Human PPP. CAT was used to evaluate the effect of JNJ9375 on thrombin generation in human PPP. In these assays, JNJ-9375 at up to $1 \mu \mathrm{M}(149 \mu \mathrm{g} / \mathrm{ml})$ demonstrated no inhibitory effect on either peak thrombin or ETP (Fig. 5, A and $\mathrm{B})$. Both endpoints increased moderately when treated with JNJ-9375, which could be partially explained by changes in the rate of thrombin inhibition by antithrombin III in the presence of JNJ-9375 (data not shown). In contrast to JNJ-9375, the active site inhibitor dabigatran produced 
A

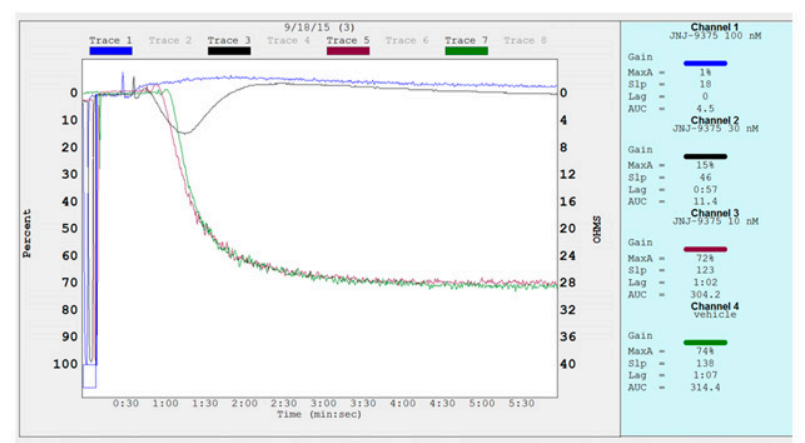

B

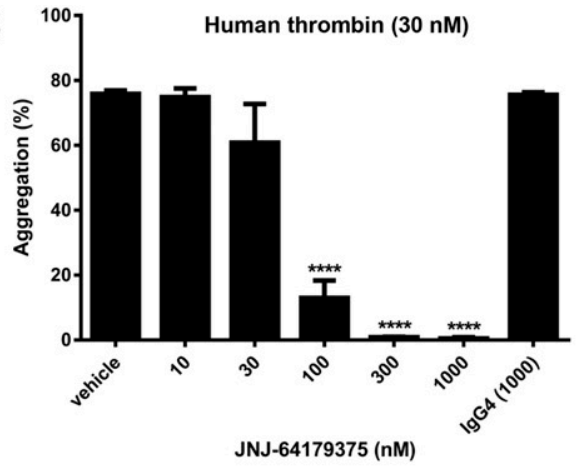

$\mathbf{E}$

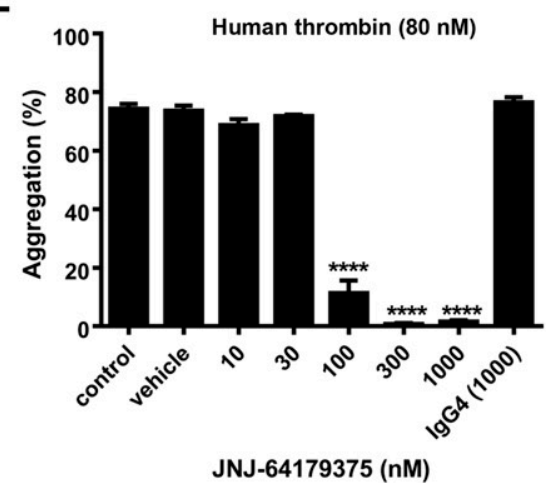

Fig. 3. (A-D) Concentration-response curves of JNJ-9375 for the prolongation of TT, ECT, PT, and aPTT in human, cynomolgus monkey, rat, mouse, and rabbit PPP; (E) concentration-response curves of JNJ-9375-induced fold prolongation of TT, ECT, PT, and aPTT in human PPP; (F) concentrationresponse curves of apixaban-induced fold prolongation of TT, ECT, PT, and aPTT in human PPP. Data are expressed as mean \pm S.E.M.; PPP obtained from four to six donors in each species.

dose-dependent inhibition of both peak thrombin and ETP (Xu et al., 2013). Only a modest delay in lag time was observed at up to $1 \mu \mathrm{M}$ JNJ-9375 (Fig. 5C). Based on these results the primary endpoints of the CAT assay (peak thrombin and ETP) are not sensitive to exosite I binding by JNJ-9375, while changes in lag time are less sensitive to JNJ-9375 than clotting assays such as TT or ECT.

Effect of JNJ-9375 on TEG in Human PPP. The anticoagulant effect of JNJ-9375 was measured in freshly drawn human citrated whole blood via TEG. JNJ-9375 was added to the blood sample and analyzed with a citrated RapidTEG protocol. JNJ-9375 produced dose-dependent prolongation of reaction time (Fig. 6), with no observed effect on maximum amplitude or fibrinolytic activity (data not shown).

Effect of JNJ-9375 on Thrombus Formation in the Rat AV Shunt Model of Thrombosis. JNJ-9375 significantly reduced thrombus weight in the rat AV shunt model of

TABLE 2

Inhibition of coagulation in PPP of different species by JNJ-9375 Data are expressed as mean; $n=4-6$ /group.

\begin{tabular}{lcccc}
\hline \multirow{2}{*}{ Species } & \multicolumn{4}{c}{$\mathrm{EC}_{2 \mathrm{X}}$} \\
\cline { 2 - 5 } & $\mathrm{TT}$ & $\mathrm{PT}$ & $\mathrm{ECT}$ & $\mathrm{aPTT}$ \\
\hline & $\mu g / m l$ & $\mu g / m l$ & $\mu g / m l$ & $\mu g / m l$ \\
Human & 4.4 & 172.6 & 12.4 & 202.7 \\
Monkey & 3 & 112.8 & 15.3 & 202.7 \\
Rat & 13.1 & 177.5 & 19.8 & 197.1 \\
Mouse & 4.2 & 258.6 & 16.4 & 575 \\
Rabbit & 6.1 & 1487.9 & 193 & N/A \\
\hline
\end{tabular}

$\mathrm{EC}_{2 \mathrm{x}}$, concentration required to double clotting time; N/A, not available. thrombosis (Fig. 7A). The thrombus weight was decreased in a dose-dependent manner from $273.4 \pm 19.3 \mathrm{mg}$ in the control group $(n=5)$ to $24.4 \pm 2.2 \mathrm{mg}$ in the $3 \mathrm{mg} / \mathrm{kg}$ group $(n=5)$, representing a $91.1 \%$ reduction at the highest dose tested in the current study. Robust efficacy was observed at $0.3 \mathrm{mg} / \mathrm{kg}$.

We measured the plasma exposure of JNJ-9375 under the current dosing regimen to assess the correlation between plasma drug levels and in vivo efficacy as well as ex vivo clotting times measurement. As shown in Fig. 7B, there was dose-dependent exposure of the drug. The mean plasma levels of JNJ-9375 were $1.68 \pm 0.13,5.04 \pm 0.36,21.34 \pm 1.93$, and $75.19 \pm 8.56 \mu \mathrm{g} / \mathrm{ml}$ at intravenous bolus doses of $0.1,0.3,1$, and $3 \mathrm{mg} / \mathrm{kg}$ JNJ-9375, respectively.

Ex vivo analysis confirmed the pharmacodynamic engagement of JNJ-9375 (Fig. 7, C-G). JNJ-9375 significantly prolonged TT by $1.5 \pm 0.1,2.5 \pm 0.2$, and $3.4 \pm 0.3$ times at intravenous bolus doses of $0.3,1$, and $3 \mathrm{mg} / \mathrm{kg}$, respectively, compared with that of the control group. TT prolongation correlated with thrombus weight reduction $\left(r^{2}=0.927\right)$. Other clotting assays including ECT, aPTT, and PT showed modest but significant prolongation by JNJ-9375 treatment.

Effect of JNJ-9375 on Bleeding Risk in a Rat Tail Transection Bleeding Model. The time to stop bleeding and total bleeding time in the vehicle-treated rats were 653.8 \pm 169.5 and $951.2 \pm 158.4$ seconds, respectively. JNJ-9375 at $3 \mathrm{mg} / \mathrm{kg}$ significantly prolonged the time to stop bleeding to $1473.8 \pm 174.8$ seconds and the total bleeding time to $1594.2 \pm$ 112.0 seconds, while 0.3 and $1 \mathrm{mg} / \mathrm{kg}$ of JNJ-9375 had no effects. The bleeding did not stop for the 30-minute observation period for the rats treated with $10 \mathrm{mg} / \mathrm{kg}$ JNJ-9375 (Fig. 8, 


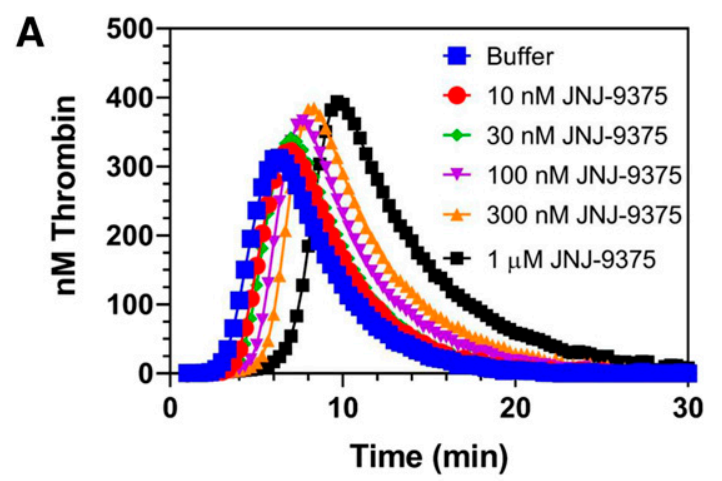

B

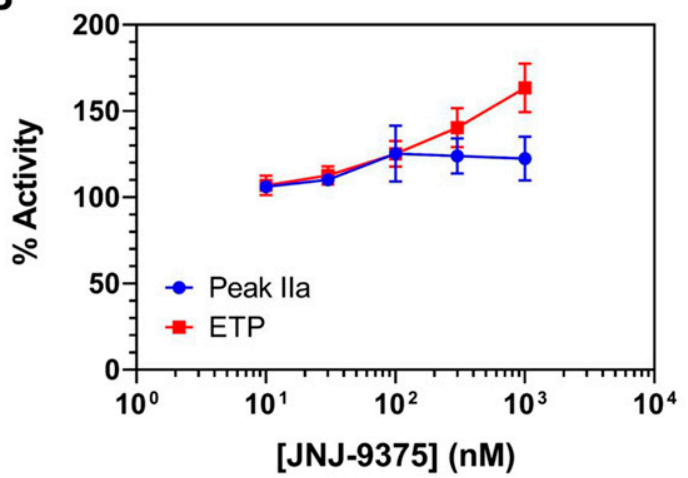

C

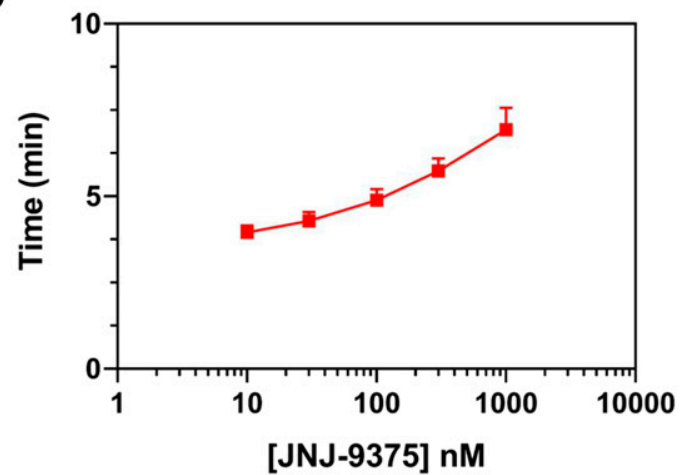

Fig. 4. Concentration response of JNJ-9375 on platelet aggregation in human PRP induced by $30 \mathrm{nM}$ human $\alpha$-thrombin: (A) representative trace; (B) dose response. (C) Lack of inhibition by JNJ-9375 on platelet aggregation in human PRP induced by ADP, TRAP-6, arachidonic acid, and collagen. Concentration response of JNJ-9375 on platelet aggregation in rat PRP induced by $60 \mathrm{nM}$ rat $\alpha$-thrombin (D) and $80 \mathrm{nM}$ human $\alpha$-thrombin (E). Data are expressed as mean \pm S.E.M.; $n=6$ /group; $* * * P<0.0001$ compared with the control group (ANOVA).

A and B). However, $10 \mathrm{mg} / \mathrm{kg}$ JNJ-9375 significantly increased the hemoglobin loss to $340.7 \pm 66.9 \mathrm{mg}$ from $90.1 \pm 29 \mathrm{mg}$ in vehicle-treated rats, while lower doses did not lead to significant hemoglobin loss (Fig. 8C).

Apixaban Effects on Thrombus Formation and Bleeding Times. The antithrombotic effects of apixaban were tested in the rat AV shunt model as a comparator, with the dose based on a previous study (Schumacher et al., 2010). Apixaban significantly reduced thrombus weight in this model, with the thrombus weight decreasing in a dosedependent manner from $246.0 \pm 9.7 \mathrm{mg}$ in the control group $(n=5)$ to $31.3 \pm 8.0 \mathrm{mg}$ in the $3 \mathrm{mg} / \mathrm{kg}$ group $(n=5)$, representing a $86.4 \%$ reduction at the highest dose tested in the current study. Robust efficacy was observed at $1 \mathrm{mg} / \mathrm{kg}$ with $57.2 \%$ reduction, which translates to the human clinical dose (based on backtranslation and modeling, data not shown)

The antithrombotic effects of apixaban were associated with a bleeding risk in our rat tail transection bleeding model. Apixaban at $1 \mathrm{mg} / \mathrm{kg}$ significantly prolonged the time to stop bleeding to $1600.7 \pm 131.6$ seconds from $513.6 \pm 31.3$ seconds in the vehicle group and the total bleeding time to $1730.0 \pm 52.2$ seconds from $765.6 \pm 103.7$ seconds in the vehicle group. Six out of the eight rats in this group did not stop bleeding for the 30-minute observation period. Hemoglobin loss significantly increased from $31.0 \pm 4.6 \mathrm{mg}$ in the vehicle group to $67.0 \pm 7.3 \mathrm{mg}$ in the $1 \mathrm{mg} / \mathrm{kg}$ apixaban group. Rats treated with $3 \mathrm{mg} / \mathrm{kg}$ apixaban did not stop bleeding in this model and hemoglobin loss further increased to $115.4 \pm 32.9 \mathrm{mg}$.

\section{Discussion}

The introduction of DOACs in the last decade has greatly improved the efficacy and safety of anticoagulant drugs. However, they still have some residual bleeding risks and as a result many patients, especially those who are at a high risk of bleeding, are underdosed or not treated at all, resulting in thrombotic events that could be prevented. Hence, there is a continued need to develop novel anticoagulants with reduced risk of bleeding.

JNJ-9375 was engineered to mimic an IgA that was found in a patient with markedly prolonged clotting times but without spontaneous bleeding episodes over a $>8$ year follow-up period, potentially representing the profile of an anticoagulant that may improve the therapeutic window with lower bleeding risk. The results of the current study demonstrate that JNJ9375, which selectively inhibits exosite I on thrombin but not the catalytic site, has antithrombotic efficacy in the rat AV shunt model in the prevention mode.

Thrombin plays a central role in the formation of hemostatic plugs and vascular thrombi by cleaving fibrinogen to form the fibrin clot and cleaving the proteinase-activated receptors to activate platelets, as well as increasing its own production via activation of factors V, VIII, and XI. When diffusing to the edge of a clot, thrombin attenuates its production by interacting with thrombomodulin on the intact endothelium cells and activates protein $\mathrm{C}$ to inhibit the Xase and prothrombinase complexes. The catalytic active site is responsible for the serine protease activity of thrombin, while anion binding exosite I mediates the interaction with substrates including 


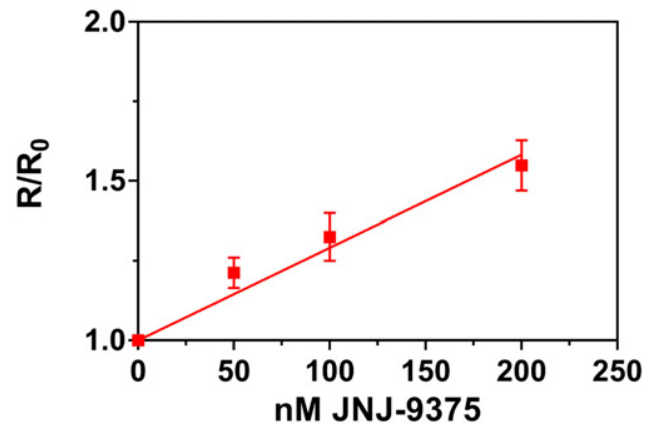

Fig. 5. Effect of JNJ-9375 on thrombin generation in human PPP initiated by recalcification in the presence of $5 \mathrm{pM}$ tissue factor and $4 \mu \mathrm{M}$ lipids: (A) representative overlaid thrombograms; (B) peak thrombin and ETP; (C) lag time. Data are expressed as mean \pm S.E.M.; PPP obtained from five different donors.

fibrinogen, proteinase-activated receptors, and thrombomodulin. Exosite II of thrombin binds to heparin and other acidic glycosaminoglycans and platelets glycoprotein $\mathrm{Ib} \alpha$. The active site and the two exosites are not available in prothrombin (Wu et al., 1994), which enables it to circulate at a high concentration when thrombin generation is needed. JNJ-9375 is a human IgG4 that binds to exosite I of thrombin from human, mouse, rat, and monkey with high affinity $\left(K_{\mathrm{D}}\right.$ value of $1-2 \mathrm{nM})$. It does not bind to prothrombin or other enzymes involved in coagulation (factors X, IX, V, and VIII), thus ensuring high specificity (data not shown). The fast $K_{\text {on }}$ value is important in a pathophysiological setting for prophylaxis, where it allows for quick neutralization of thrombin that is generated in an explosive manner locally at sites of vascular injury.

JNJ-9375 prolonged coagulation times in PPP from all of the different species tested in a concentration-dependent manner, with TT being the most sensitive assay, followed by ECT, and then at much higher concentrations PT and aPTT, as demonstrated by the calculated $\mathrm{EC}_{2 \mathrm{X}}$ value. Thus, TT and ECT could be potential assays to evaluate the pharmacodynamics of JNJ9375 since both directly determine the engagement of thrombin. Thrombin is the most potent physiologic agonist to induce platelet activation and aggregation when generated on the platelet surface during a hemostatic event. JNJ-9375 inhibited platelet aggregation induced by thrombin but not by other common agonists, confirming the specificity. JNJ9375 inhibited thrombin but not PAR1 agonist TRAP6-induced aggregation, consistent with the exosite I mode of action. The in vitro antiplatelet activity of JNJ-9375 is consistent with reports on previous thrombin exosite I inhibitors (Kelly et al., 1992; Schumacher et al., 1993; Muramatsu et al., 1997).

CAT and TEG assays were employed to evaluate the anticoagulant properties of JNJ-9375 beyond standard clotting assays. Both assays offer global coagulation profiles in plasma (CAT) or whole blood (TEG), encompassing both the pro- and antithrombogenic phases of hemostasis, and have been previously used to evaluate multiple classes of thrombin inhibitors (Xu et al., 2013). The CAT assay displayed a remarkable lack of sensitivity toward JNJ-9375, with no discernable inhibitory effect on peak thrombin or ETP at concentrations up to $1 \mu \mathrm{M}$. This lack of effect is likely because JNJ-9375 has no appreciable impact on cleavage of the CAT assay's peptidyl reporter substrate. In contrast, in TEG assays, JNJ-9375 produced a concentration-dependent increase in reaction time while having negligible effect on the other primary TEG endpoints such as maximum amplitude or fibrinolysis.

To assess antithrombotic efficacy in vivo, we used the rat AV shunt model of thrombosis, which has been well validated and
A

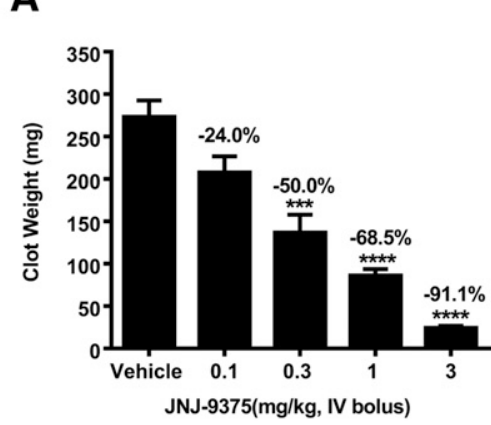

B

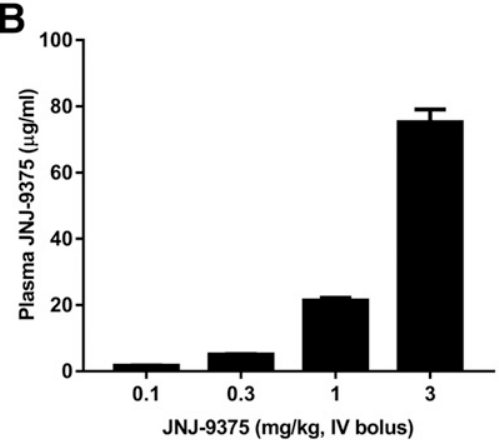

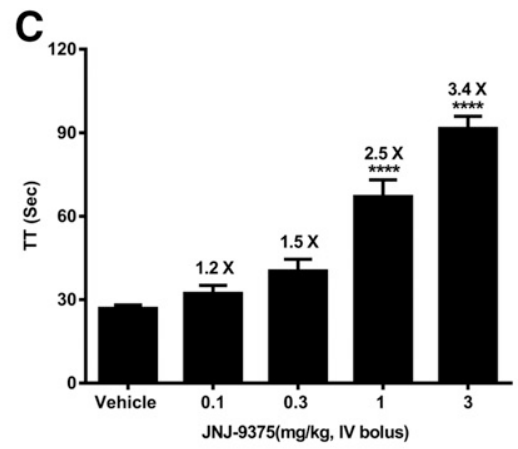

$\mathbf{F}$

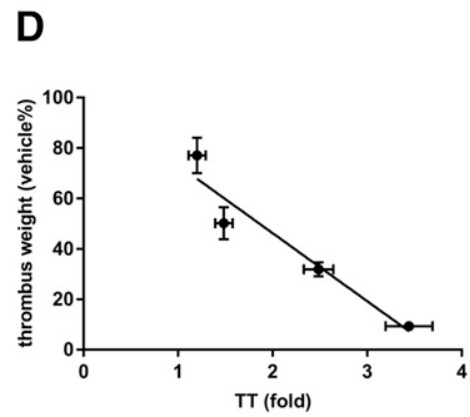

E

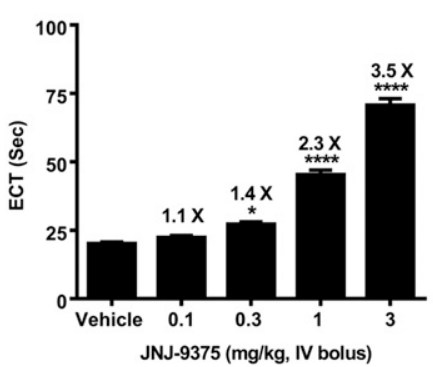

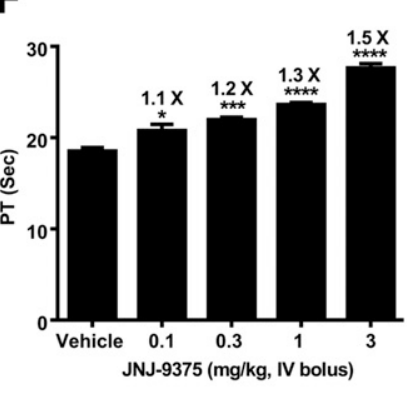

G

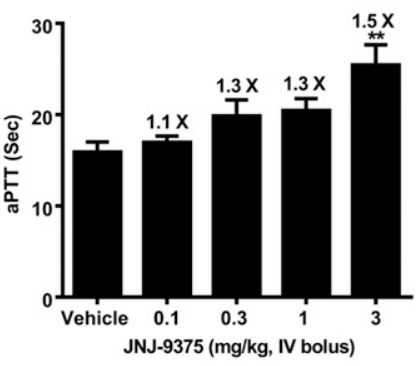

Fig. 6. Effect of JNJ-9375 on clotting reaction times (R) in human whole blood evaluated using a modification of the Citrated RapidTEG TEG protocol. The fold increase in reaction time compared with buffer control $\left(\mathrm{R} / \mathrm{R}_{0}\right)$ is plotted vs. JNJ-9375 concentration. Data are expressed as mean \pm S.E.M.; sample obtained from three donors. 

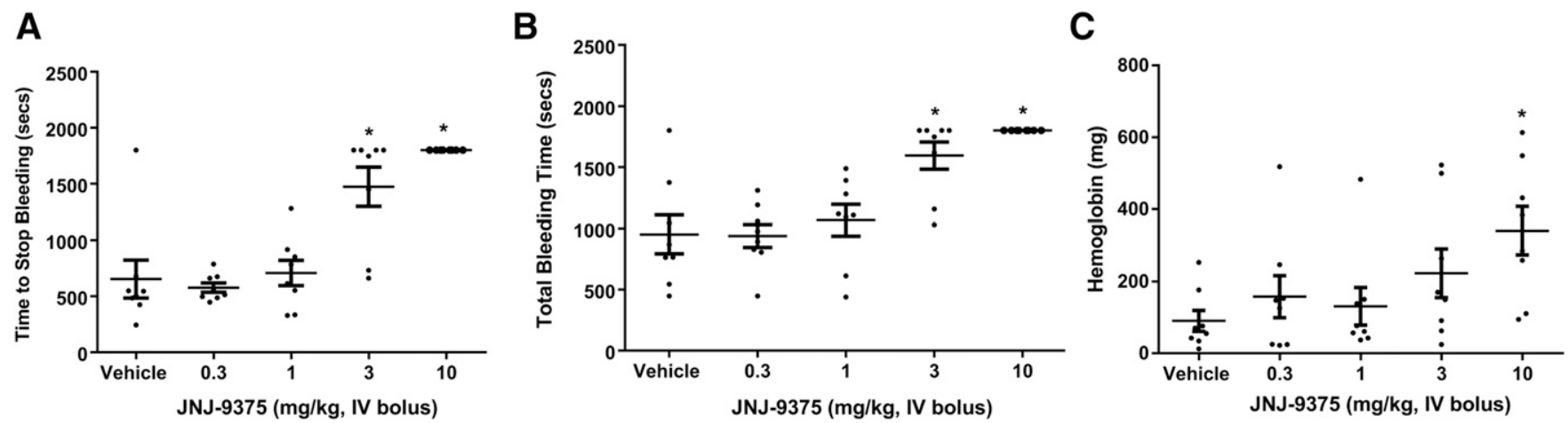

Fig. 7. (A) Dose response of JNJ-9375 in the rat AV shunt model of thrombosis. (B) JNJ-9375 plasma exposure. (C) Prolongation of TT in ex vivo plasma samples. (D) Correlation between TT prolongation and decrease in thrombus weight. (E-G) ECT, PT, and aPTT in ex vivo plasma samples, respectively. Data are expressed as mean \pm S.E.M.; $n=5 /$ treatment group. ${ }^{*} P<0.05 ;{ }^{*} P<0.01 ; * * * P<0.001 ;{ }^{* * * *} P<0.0001$ compared with the vehicle group (one-way ANOVA).

widely used (Leadley et al., 2000). Under the current experimental conditions, JNJ-9375 achieved robust antithrombotic efficacy at the $0.3 \mathrm{mg} / \mathrm{kg}$ dose with an approximately 1.5 -fold increase in TT and ECT. The pharmacokinetic analyses demonstrated a consistent dose-related trend between plasma drug exposure and in vivo efficacy as well as ex vivo clotting parameters. These data suggest that selective inhibition of exosite I on thrombin leads to antithrombotic activity. Hirugen, a synthetic peptide that inhibits the exosite I of thrombin, but much more weakly than JNJ-9375 (the $\mathrm{EC}_{2 \mathrm{X}}$ value for TT was $1 \mu \mathrm{M}$ vs. $29 \mathrm{nM}$ for JNJ-9375), still substantially decreased fibrin deposition in a baboon AV shunt model under low shear conditions (Kelly et al., 1992). BMS 180742, another exosite I-specific inhibitor that has a sequence similar to the C-terminal peptide sequence of hirudin, a bivalent direct thrombin inhibitor that binds both the catalytic site and exosite I, inhibited thrombus formation in a dose-dependent fashion in a rat vena cava venous thrombosis model (Seiler et al., 1991; Schumacher et al., 1993). These data collectively demonstrate that inhibition of thrombin exosite $\mathrm{I}$ is an effective antithrombotic mechanism in preclinical animal models, at least in a prevention mode.

In our rat tail transection bleeding model, JNJ-9375 significantly increased bleeding at 3 and $10 \mathrm{mg} / \mathrm{kg}$, as illustrated by significant increased time to stop bleeding and total bleeding time. JNJ-9375 mildly increased blood loss at $10 \mathrm{mg} / \mathrm{kg}$ as measured by hemoglobin loss. Since JNJ-9375 reduced thrombus weight by $50 \%$ at a dose of $0.3 \mathrm{mg} / \mathrm{kg}$ in the AV shunt model, the therapeutic index was $10 \times$. Apixaban inhibited thrombus weight by $50 \%$ at a dose of $1 \mathrm{mg} / \mathrm{kg}$, while in the rat tail transection bleeding model apixaban induced significant bleeding at a dose of $1 \mathrm{mg} / \mathrm{kg}$. The therapeutic index for apixaban was $1 \mathrm{X}$ in our models, which is consistent with the results from a prior publication in rats (Schumacher et al., 2010). The different bleeding profile of JNJ-9375 is consistent with the lack of bleeding episode in the index patient despite the abnormal clotting times, which could potentially be due to the inability of JNJ-9375 to penetrate a clot, the thrombin active site sparing effect, or the relative impacts on the prothrombotic (fibrinogen and PAR1) and antithrombotic (thrombomodulin) pathways of thrombin. The mechanistic insights are worth further evaluation.

Based on these preclinical data, JNJ-9375 was advanced into clinical development. However, despite the promising preclinical animal efficacy and safety data, in a recently completed phase 2 randomized double-blind trial, JNJ-9375 was safe but less effective than apixaban in preventing thrombosis in patients undergoing knee replacement surgery when given postoperatively at doses of $0.1-1.8 \mathrm{mg} / \mathrm{kg}$, i.v. (Weitz et al., 2019).The reason for the lack of efficacy is not clear, since the pharmacodymanic marker such as TT, a marker of anticoagulant activity, was elevated in a dosedependent manner. One possibility is that exosite 1 inhibition on thrombin in humans may not be an effective antithrombotic approach compared with what we have observed in rats. Another possibility is that in all of the preclinical studies, JNJ-9375 was administered prior to initiation of thrombus in a prevention mode versus a treatment mode in the phase II study. It is not clear if the drug has the same efficacy profile in a treatment mode. One cannot rule out the possibility that the size limitation of the antibody to penetrate and attenuate the growth of preformed thrombi may be different from a prevention setting. In this context, it was shown that a p-selectin antibody was unable to label all activated platelets in a thrombus when administered after clot formation but did so when administered prior to clot formation (Stalker et al., 2013). Last but not least, in the AV shunt model, the thrombus is initiated on an artificially introduced silk thread due to the contact activation of the coagulation and potentially to some platelets. This could be different from the pathophysiology of human venous thrombotic diseases, which likely involves an intricate interplay between sheer, blood flow, anatomic structure, endothelium activation, and vasoconstriction. The fundamental differences may account for the inability to translate the preclinical animal model data to clinical efficacy. It is possible that higher doses of JNJ-9375 may be required in humans than those required in animals; however, we observed increased bruising at the injection sites at doses of $2.5 \mathrm{mg} / \mathrm{kg}$ in healthy volunteers in our phase I clinical trials.

In conclusion, JNJ-9375 is a novel, recombinant human IgG4 monoclonal antibody that binds specifically to the exosite I region of thrombin with high affinity. We have demonstrated robust in vitro and ex vivo anticoagulant activity and in vivo antithrombotic efficacy, as well as reduced bleeding risk compared with apixaban, which support the antithrombotic effects of JNJ-9375 (namely, the mechanism of inhibiting the thrombin exosite I) in the rat $\mathrm{AV}$ shunt model in a prevention mode. However, JNJ-9375 was not as effective in preventing 
thrombus formation compared with apixaban in patients undergoing total knee replacement surgery.

\section{Acknowledgments}

We thank Peter Garret and Brian Geist (Biologics Development Sciences, Janssen Research \& Development LLC) for measuring the JNJ-9375 plasma concentration and Bin Gao (Quantitative Sciences, Janssen Research \& Development LLC) for statistical analysis.

\section{Authorship Contributions}

Participated in research design: Devine, Bunce, Chintala.

Conducted experiments: Devine, Du, Li, Bunce, Lacy.

Performed data analysis: Devine, Du, Li, Bunce, Lacy, Chintala.

Wrote or contributed to the writing of the manuscript: Devine, Bunce, Lacy, Chintala.

\section{References}

Baglin TP, Langdown J, Frasson R, and Huntington JA (2016) Discovery and characterization of an antibody directed against exosite I of thrombin. $J$ Thromb Haemost 14:137-142

Barra ME, Fanikos J, Connors JM, Sylvester KW, Piazza G, and Goldhaber SZ (2016) Evaluation of dose-reduced direct oral anticoagulant therapy. Am J Med 129: 1198-1204.

Bradford HN and Krishnaswamy S (2012) Meizothrombin is an unexpectedly zymogen-like variant of thrombin. J Biol Chem 287:30414-30425.

Derian CK, Santulli RJ, Tomko KA, Haertlein BJ, and Andrade-Gordon P (1995) Species differences in platelet responses to thrombin and SFLLRN. receptormediated calcium mobilization and aggregation, and regulation by protein kinases. Thromb Res 78:505-519.

Douxfils J, Mullier F, Robert S, Chatelain C, Chatelain B, and Dogné JM (2012) Impact of dabigatran on a large panel of routine or specific coagulation assays. Laboratory recommendations for monitoring of dabigatran etexilate. Thromb Haemost 107:985-997.

Eisert WG, Hauel N, Stangier J, Wienen W, Clemens A, and van Ryn J (2010) Dabigatran: an oral novel potent reversible nonpeptide inhibitor of thrombin Arterioscler Thromb Vasc Biol 30:1885-1889.

Furie B and Furie BC (2008) Mechanisms of thrombus formation. N Engl J Med 359: 938-949.

Hawes BE, Zhai Y, Hesk D, Wirth M, Wei H, Chintala M, and Seiffert D (2015) In vitro pharmacological characterization of vorapaxar, a novel platelet thrombin receptor antagonist. Eur J Pharmacol 762:221-228.

Huntington JA (2013) Thrombin inhibition by the serpins. J Thromb Haemost 11 (Suppl 1):254-264.

Kelly AB, Maraganore JM, Bourdon P, Hanson SR, and Harker LA (1992) Antithrombotic effects of synthetic peptides targeting various functional domains of thrombin. Proc Natl Acad Sci USA 89:6040-6044.

Leadley RJ Jr, Chi L, Rebello SS, and Gagnon A (2000) Contribution of in vivo models of thrombosis to the discovery and development of novel antithrombotic agents. J Pharmacol Toxicol Methods 43:101-116.

Muramatsu R, Sasaki M, Watanabe N, Goto Y, Okayama T, Nukui E, Morikawa T, and Hayashi H (1997) Antithrombotic effects of NE-6505, a novel anion-binding exosite inhibitor. Thromb Res 86:453-460.

Ruff CT; Giugliano RP, Braunwald E, Hoffman EB, Deenadayalu N, Ezekowitz MD, Camm AJ, Weitz JI, Lewis BS, Parkhomenko A, Yamashita T, Antman EM (2014)
Comparison of the efficacy and safety of new oral anticoagulants with warfarin in patients with atrial fibrillation: a meta-analysis of randomised trials. Lancet 383 : 955-962.

Sambrano GR, Weiss EJ, Zheng YW, Huang W, and Coughlin SR (2001) Role of thrombin signalling in platelets in haemostasis and thrombosis. Nature 413: $74-78$.

Schumacher WA, Bostwick JS, Stewart AB, Steinbacher TE, Xin B, and Wong PC (2010) Effect of the direct factor Xa inhibitor apixaban in rat models of thrombosis and hemostasis. J Cardiovasc Pharmacol 55:609-616.

Schumacher WA, Steinbacher TE, Heran CL, Seiler SM, Michel IM, and Ogletree ML (1993) Comparison of thrombin active site and exosite inhibitors and heparin in experimental models of arterial and venous thrombosis and bleeding. J Pharmacol Exp Ther 267:1237-1242.

Seiler SM, Goldenberg HJ, Michel IM, Hunt JT, and Zavoico GB (1991) Multiple pathways of thrombin-induced platelet activation differentiated by desensitization and a thrombin exosite inhibitor. Biochem Biophys Res Commun 181:636-643.

Shang J, Chen Z, Wang M, Li Q, Feng W, Wu Y, Wu W, Graziano MP, and Chintala M (2014) Zucker diabetic fatty rats exhibit hypercoagulability and accelerated thrombus formation in the arterio-venous shunt model of thrombosis. Thromb Res 134:433-439.

Stalker TJ, Traxler EA, Wu J, Wannemacher KM, Cermignano SL, Voronov R, Diamond SL, and Brass LF (2013) Hierarchical organization in the hemostatic response and its relationship to the platelet-signaling network. Blood 121: $1875-1885$

Turpie AG; Haas S, Kreutz R, Mantovani LG, Pattanayak CW, Holberg G, Jamal W, Schmidt A, van Eickels M, Lassen MR (2014) A non-interventional comparison of rivaroxaban with standard of care for thromboprophylaxis after major orthopaedic surgery in 17,701 patients with propensity score adjustment. Thromb Haemost 111:94-102.

Weitz JI and Harenberg J (2017) New developments in anticoagulants: past, present and future. Thromb Haemost 117:1283-1288.

Weitz JI and Jaffer IH (2016) Optimizing the safety of treatment for venous thromboembolism in the era of direct oral anticoagulants. Pol Arch Med Wewn 126: 688-696.

Weitz JI, Raskob G, Lassen MR, Segers A, Francis C, Roberts R, Fuji T, Peters G, Swaim R, Johnson M, et al. (2019) Comparison of JNJ9375, a thrombin exosite 1directed antibody, with apixaban for thromboprophylaxis after elective knee replacement surgery: TEXT-TKR study. J Am Coll Cardiol 73:2047.

Wu Q, Picard V, Aiach M, and Sadler JE (1994) Activation-induced exposure of the thrombin anion-binding exosite. Interactions of recombinant mutant prothrombins with thrombomodulin and a thrombin exosite-specific antibody. J Biol Chem $\mathbf{2 6 9}$ 3725-3730.

Xian Y, O'Brien EC, Liang L, Xu H, Schwamm LH, Fonarow GC, Bhatt DL, Smith EE, Olson DM, Maisch L, et al. (2017) Association of preceding antithrombotic treatment with acute ischemic stroke severity and in-hospital outcomes among patients with atrial fibrillation. JAMA 317:1057-1067.

Xu Y, Wu W, Wang L, Chintala M, Plump AS, Ogletree ML, and Chen Z (2013) Differential profiles of thrombin inhibitors (heparin, hirudin, bivalirudin, and dabigatran) in the thrombin generation assay and thromboelastography in vitro. Blood Coagul Fibrinolysis 24:332-338.

Zhou L and Schmaier AH (2005) Platelet aggregation testing in platelet-rich plasma: description of procedures with the aim to develop standards in the field. Am J Clin Pathol 123:172-183.

Address correspondence to: Zheng Huang Devine, Cardiovascular \& Metabolism, Janssen Research \& Development LLC, 1400 McKean Road, Spring House, PA 19477. E-mail: zhuangde@ITS.JNJ.com 